\title{
LARS2-Perrault syndrome: a new case report and literature review
}

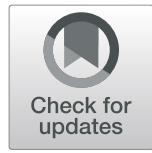

\author{
Maria Teresa Carminho-Rodrigues ${ }^{1 *}$, Phillipe Klee ${ }^{2}$, Sacha Laurent ${ }^{1}$, Michel Guipponi ${ }^{1}$, Marc Abramowicz ${ }^{1}$ \\ Hélène Cao-van ${ }^{3}$, Nils Guinand ${ }^{3}$ and Ariane Paoloni-Giacobino ${ }^{1}$
}

\begin{abstract}
Background: Perrault syndrome is a rare recessive and genetically heterogeneous disorder characterized by sensorineural hearing loss in males and females and gonadal dysgenesis in females. Mutations in seven different genes have been identified: HARS2, HSD17B4, CLLP, C10orf, ERAL1, TWNK and LARS2.

To date, 19 variants have been reported in 18 individuals with LARS2-Perrault syndrome.

Case presentation: Here we describe the case of an 8-year-old girl with compound heterozygous missense mutations in the LARS2 gene. We identified two missense mutations [c.457A > C, p.(Asn153His) and c.1565C > A, p.(Thr522Asn)] and subsequent familial segregation showed that each parent had transmitted a mutation.

Conclusions: These results have implications for genetic counseling and provide insight into the functional role of LARS2. This case highlights the importance of an early diagnosis. Systematic genetic screening of children with hearing loss allows the early identification of a Perrault syndrome in order to ensure specific endocrinological surveillance and management to prevent secondary complications. Clinical data are compared with the other cases reported in the literature.
\end{abstract}

Keywords: Perrault syndrome, LARS2, Whole-exome sequencing, Sensorineural hearing loss

\section{Background}

Perrault syndrome (MIM: 233400) is a rare recessive genetically heterogeneous disorder characterized by sensorineural hearing loss in males and females and ovarian dysfunction in females [1]. One-third of all patients have mutations in one of the seven known causative genes: HARS2 (MIM 600783), HSD17B4 (MIM 601860) CLPP (MIM 601119), C10orf2, (MIM606075), ERAL1 (MIM 607435), TWNK (MIM 606075) and LARS2 (MIM 604544) [2].

More recently Tracewska-Siemiatkowska A et al., 2017 described a girl with a profound congenital hearing impairment and primary amenorrhea, like in Perrault Syndrome that also present additional features like

\footnotetext{
* Correspondence: mcih@hcuge.ch

'Department of Genetic Medicine, University Hospitals of Geneva Rue, Gabrielle-Perret-Gentil 4, 1211 Genève 14, Switzerland

Full list of author information is available at the end of the article
}

progressive retinal degeneration, agenesis of the corpus callosum, and liver disease. A homozygous variant in YARS (MIM 603623), a gene previously related with Charcot-Marie-Tooth disease, was identified [3].

LARS2 encodes mitochondrial leucyl-tRNA synthetase (mtLeuRS), a 903 amino acid protein [4, 5]. AminoacyltRNA synthetases then attach specific amino acids to the $3^{\prime}$ ends of their cognate tRNAs, which is required in the cytoplasm and mitochondria for the translation of nuclear and mitochondrial encoded genes, respectively. Apart from two aminoacyl transfer RNA synthases, glycyl-tRNA synthetase and lysyl-tRNA synthetase, all other synthetases are encoded by separate genes for nuclear and mitochondrial functions [6]. However, the human structure of mtLeuRS has not been elucidated.

Apart from hearing loss and ovarian dysgenesis in females, some patients with Perrault syndrome may present neurological symptoms, such as learning 
disabilities, pyramidal signs, cerebellar ataxia and motor or sensory peripheral neuropathy [7]. Here, we report the case of a young girl with bilateral severe hearing loss and bi-allellic mutations in LARS2.

\section{Case presentation}

Our subject was an 8-year-old girl born at 36 weeks' gestation and the first child of healthy non-consanguineous French parents. Family history was unremarkable. She has one healthy younger brother. Her birth weight was 2200 g. Newborn hearing screening was not performed at birth. Developmental milestones were normal with walking at the age of 16 months and first words at 12 months. At the age of 4 years, she started to present difficulties at nursery school that led to hearing screening. A bilateral severe sensorineural hearing loss was finally identified at the age of 7 years. Tonal audiograms (Fig. 1) did not show the upsloping pattern considered as typical for HARS2 mutations and described in one case of a LARS2 mutation [8]. Hearing loss was severe on both sides with a discreet $\mathrm{U}$-shaped curve centered on the $1000 \mathrm{~Hz}$ frequency. Transient otoacoustic emission and distortion product otoacoustic emission of both ears were absent. Auditory evoked potentials showed recognized curves at $80 \mathrm{~dB}$ on both sides and confirmed severe hearing loss. A temporal bone computed tomography scan and magnetic resonance imaging (MRI) performed to exclude other inner ear malformations were normal.

At the age of 7 years, she received a cochlear implant on the right side (CI-522 cochlear) and responded well. One year later, the tonal free field audiogram showed 25 $\mathrm{dB}$ hearing levels on conversational frequencies and the vocal audiogram showed $100 \%$ of comprehension at 60 $\mathrm{dB}$ (intensity of normal voice). She had no neurological symptoms and her cognitive development was normal. At physical examination, she exhibited normal growth parameters and no dysmorphic features. She had astigmatism and three small café-au-lait macules. At the age of 8 years and 7 months, an endocrinological workup showed a chronological bone age that predicted a normal adult height. Hormonal assessments showed normal prepubertal values of follicle-stimulating hormone (FSH) $(2.8 \mathrm{IU} / \mathrm{L})$ and luteinizing hormone $(\mathrm{LH})(0.7 \mathrm{U} / \mathrm{L})$. Estradiol was below the detection limit of $17 \mathrm{pmol} / \mathrm{l}$. Inhibin $\mathrm{B}$ was undetectable and the anti-Mullerian hormone $(\mathrm{AMH})$ was $1.0 \mathrm{pmol} / \mathrm{l}$. A pelvic ultrasound showed a normal prepubertal uterus measuring $32 \mathrm{~mm}$

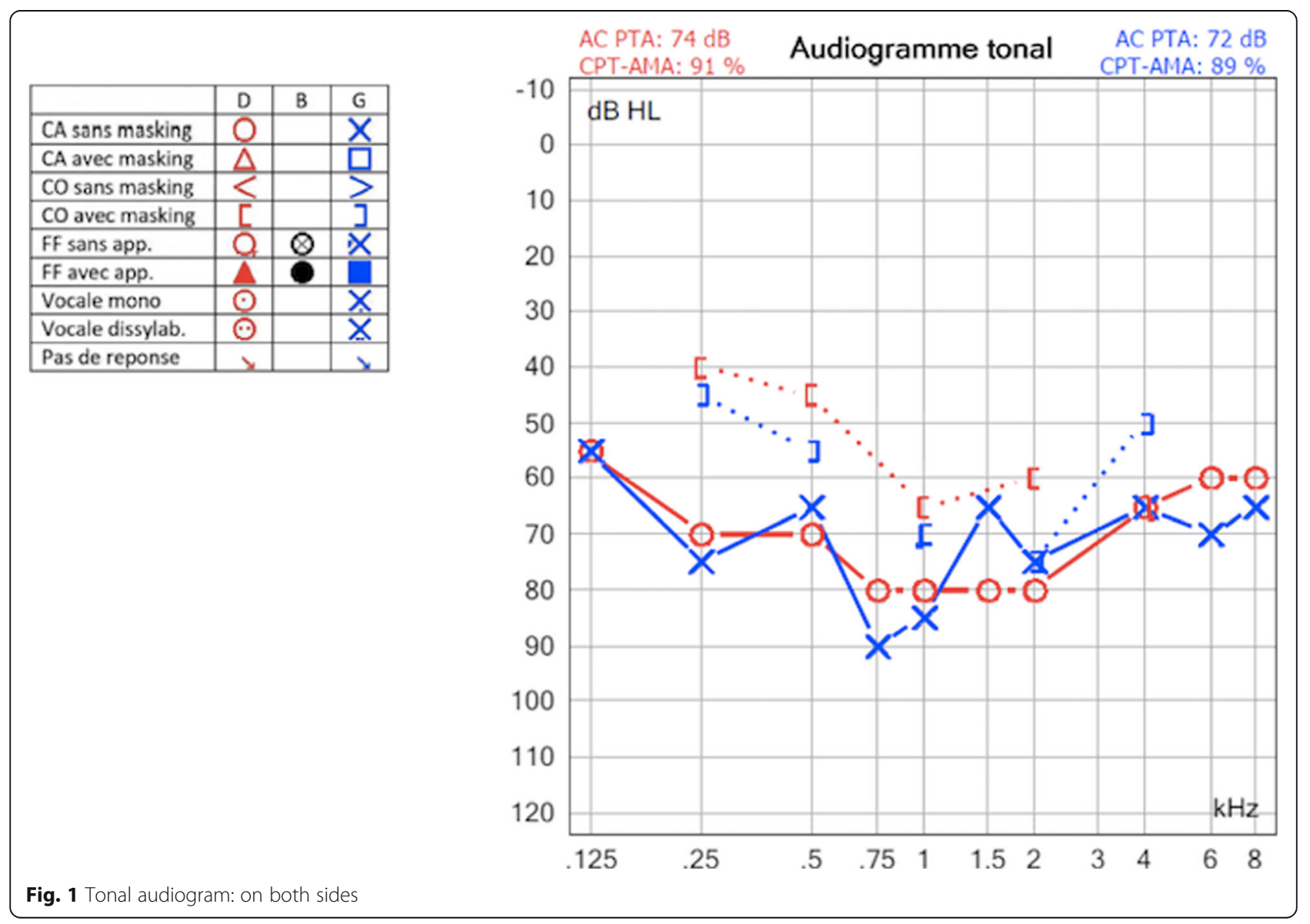


in length. A right ovary measuring $16 \times 6 \times 6 \times 6.5 \mathrm{~mm}$ was visualized. The left ovary was not clearly visualized. Thyroid function, insulin-like growth factor 1 and morning cortisol values $(275 \mathrm{nmol} / \mathrm{l})$ were normal. Glucose metabolism was normal with fasting blood glucose values of $4.7 \mathrm{mmol} / \mathrm{l}$, insulin, $3.3 \mathrm{mIU} / \mathrm{l}$, and glycated haemoglobin, $5.0 \%(31.0 \mathrm{mmol} / \mathrm{mol})$.

\section{Molecular investigation}

Written informed consent from the parents was obtained prior to molecular studies and whole-exome sequencing was performed. Exome of the patient was captured using the SureSelet QXT Human All Exon V5 kit (Agilent Technologies Inc., Santa Clara, CA, USA) and sequenced on a NextSeq500 instrument (Illumina, San Diego, CA, USA).

Reads mapping and variant calling were performed using BWA 0.7.13, Picard 2.9.0 and GATK HaplotypeCaller 3.7 and annotated with annovar 2017-07-17 and UCSC RefSeq (refGene) downloaded on 2018-08-10. The variants were searched for in various databases including dbSNP151, gnomAD 2.1, ClinVar 2018 and HGMD 2016.

Pathogenicity prediction scores were obtained for missense variants using SIFT, PolyPhen, MutationTaster and CADD. Splicing effect alterations were assessed using dbscSNV.

Our patient was found to carry two missense variants in the LARS2 gene (NM_015340.3.3): c.457A $>$ C, p.(Asn153His) in exon 6 rs786205560, and c.1565C > A, p.(Thr522Asn) in exon 14, rs199589947. Segregation analysis showed that the patient inherited the p.(Asn153His) variant from her father and p.(Thr522Asn) from her mother, consistent with an autosomal recessive mode of inheritance.

The c.457A $>$ C, p.(Asn153His) variant is absent from the gnomAD database and has been classified once in ClinVar as "likely pathogenic" and once as "of uncertain clinical significance". The amino acid change was predicted as pathogenic by all used algorithms. As the c.457 A $>\mathrm{C}$ variant changed the second nucleotide of exon 6 , it could therefore have affected splicing, as predicted by the dbscSNV (score $=0.93$ ) and recently reported in homozygosity by Al-Jaroudi et al. [9] The c. $1565 \mathrm{C}>\mathrm{A}, \mathrm{p}$.(Thr522Asn) variant is present in the gnomAD database $\left(\mathrm{MAF}=2.8 \times 10^{-4}\right)$ at a frequency compatible with a recessive mode of inheritance. It is also predicted as pathogenic by all used algorithms and is classified three times as "pathogenic" and once as "likely pathogenic" in ClinVar. It has already been reported in the literature as associated with Perrault syndrome $[8,10]$. According to the ACMG guidelines [11, 12], we classified the p.(Asn153His) and p.(Thr522Asn) variants as pathogenic. The presence of these two variants in compound heterozygosity indicated that our patient presented a Perrault syndrome.

\section{Discussion and conclusions}

We described a young girl with a Perrault syndrome caused by a bi-allelic LARS 2 mutations. To our knowledge, our patient is only the second case of a p.Asn153His Perrault syndrome reported in the literature and the first case reported in compound heterozygosity. The first case reported with p.Asn153His in homozygosity was a 27- year-old female with Perrault syndrome type 1 [9]. Regarding the second mutation, p.Thr522Asn, has been already described several times. It has also been implicated in a LARS2 more severe phenotype described by Riley et al. [13] They described a lethal, multisystem metabolic disorder characterized by severe lactic acidosis, hydrops and sideroblastic anemia, impaired cardiac function, disordered coagulation, pulmonary hypertension and progressive renal disease associated with the biallelic LARS2 p.Thr522Asn and p.Ala430Val mutations $[7,11]$. Thus, the presence of p.Thr522Asn in our patient reinforced the idea that an association with the p.Ala430Val variant would have been more likely to be more damaging than p.Thr522Asn already described in homozygosity in Perrault syndrome $[8,10]$.

The identification of two missense variants both previously reported in patients with Perrault syndrome is in full agreement with all previous variants being of missense type, and it has been proposed that inactivating variants in these essential genes, may not be compatible with life.

To date, 19 variants have been identified in LARS2 in 18 individuals with Perrault syndrome (Table 1). A comparison of the literature showed that bilateral hearing loss was highly variable, ranging from moderate to profound, and sometimes progressive. Of note, all individuals with a p.Thr522Asn Perrault syndrome, either homozygous or compound heterozygous, presented with sensorineural hearing loss, which was worse at low frequencies [5, 10]. Age of onset also varied between 18 months and 32 years, but it was difficult to evaluate as detailed information was not always available.

Amenorrhea was most frequently primary and there appeared to be no obvious genotype-phenotype correlation [15].

It is still not clear if LARS2 can be responsible for an isolated hearing loss in the case of our young female patient and other prepubertal female patients described before. Follow up is essential to elucidate this question.

Neurological symptoms were present in five families with neurodevelopmental delay, gait ataxia, behavioral problems and pyramidal dysfunction [7, 15]. Van der Knaap et al. [15] reported three patients who developed 


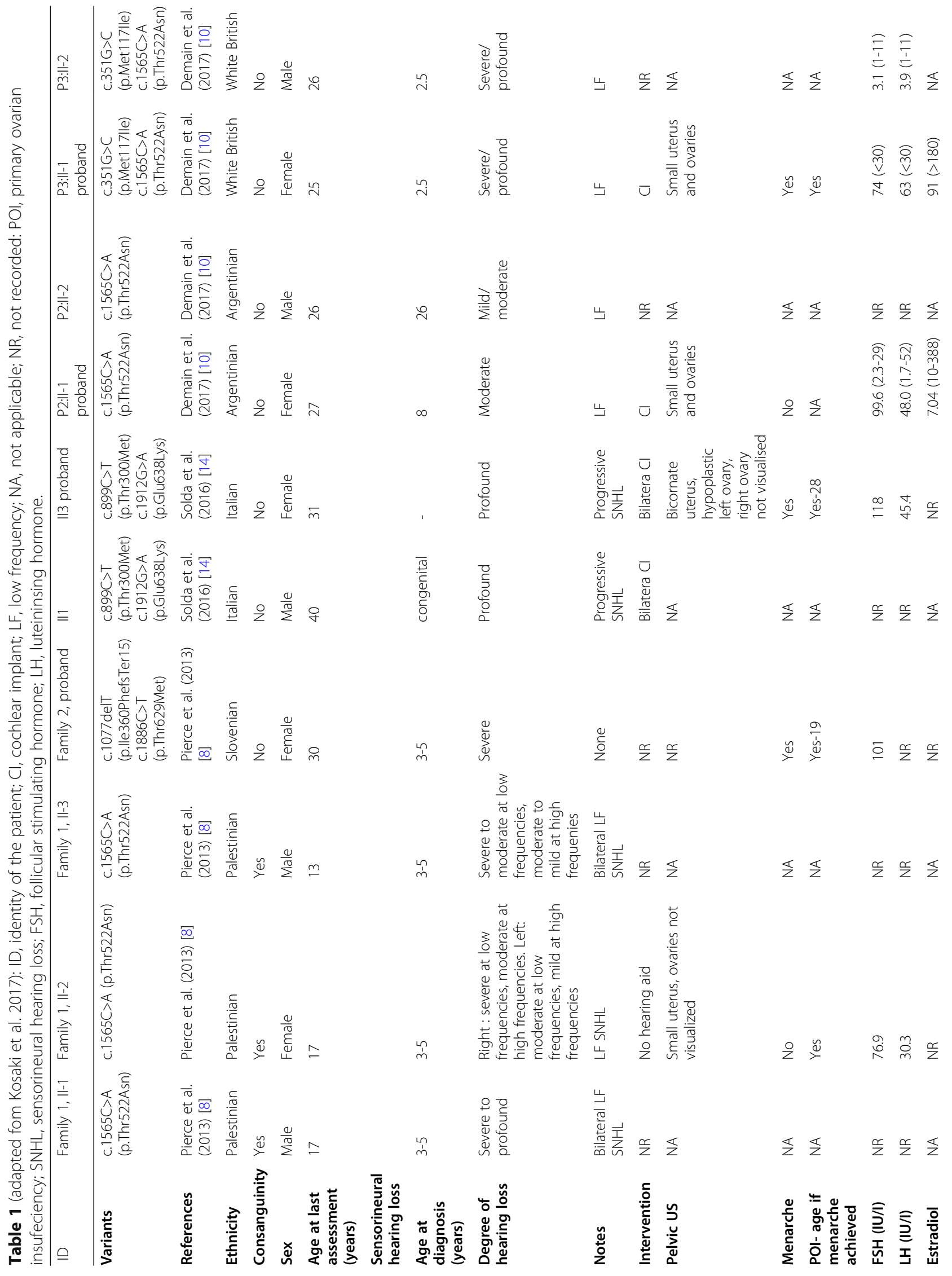




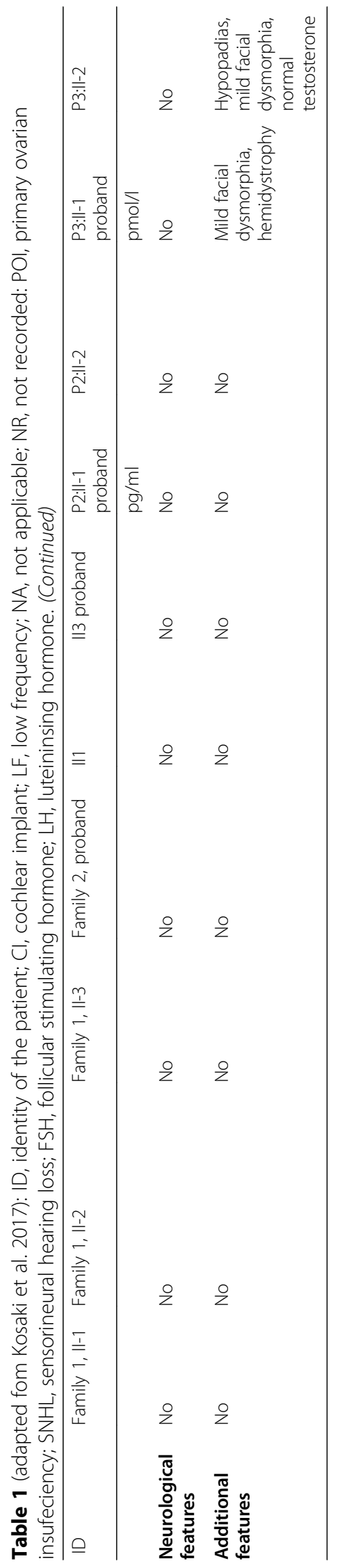




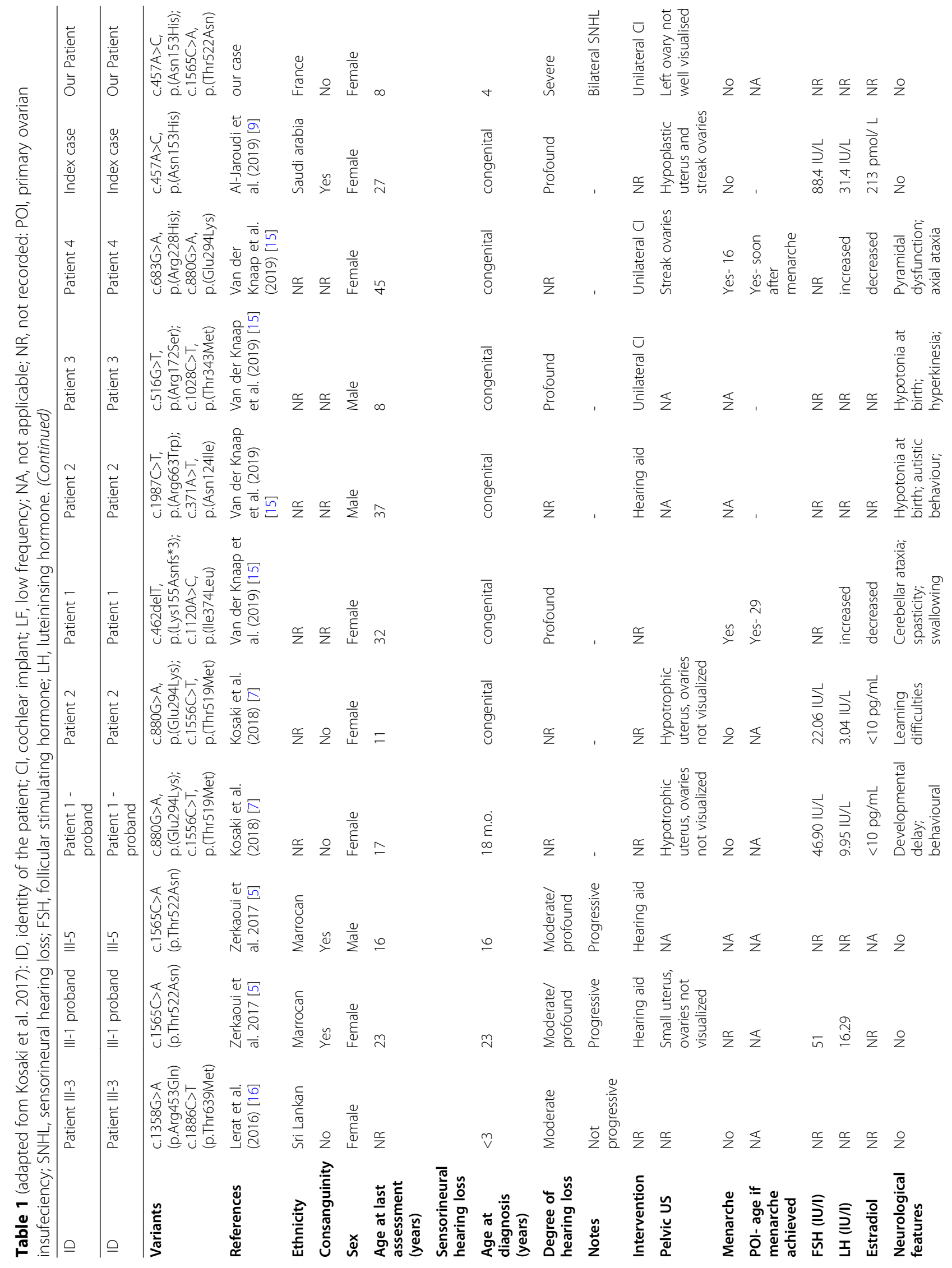




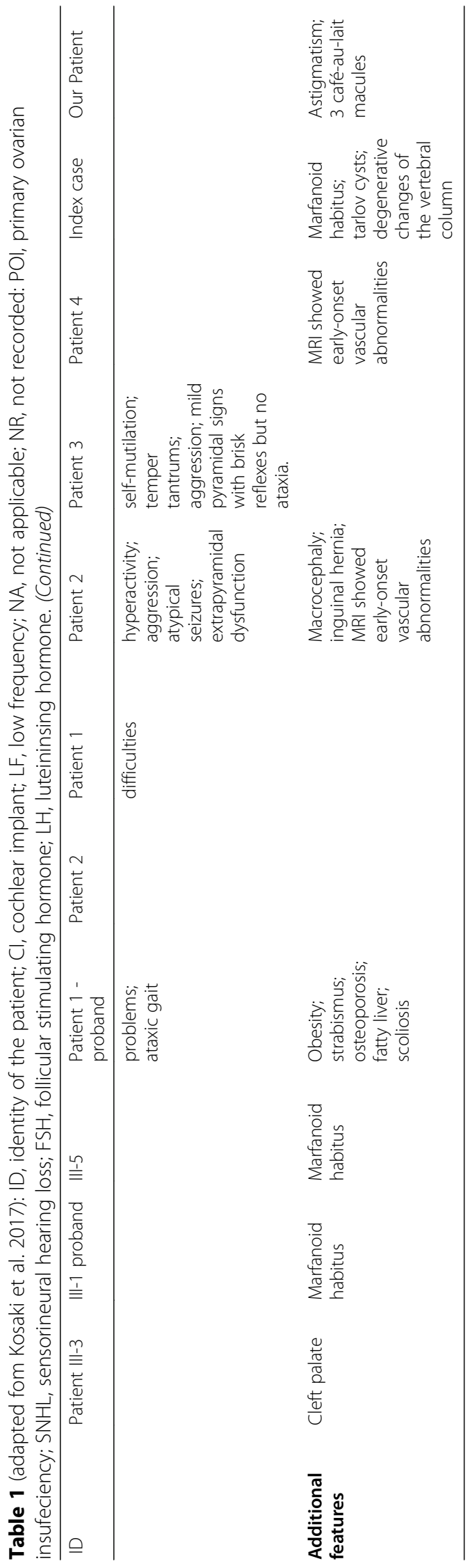


severe neurological features in later life. Cerebral MRIs performed at adult age showed extensive white matter abnormalities and additional signs of early-onset vascular abnormalities were observed in two patients [13]. However, as most patients were relatively young at genetic diagnosis, it remains unclear whether neurological symptoms may develop later [5].

In our patient, the fact that the diagnosis was made at an early age was crucial for this young girl. She was referred to the pediatric endocrinologist for evaluation and follow- up. Endocrinologic work-up showed normal prepubertal values for FSH, LH and estradiol. A pelvic ultrasound showed a normal prepubertal uterus and allowed visualization of a right gonad. Visualization of the left gonad was difficult. Inhibin B and AMH were measured at a time point where both hormones are naturally low: inhibin B was undetectable and $A M H$ was below normal values. While visualization of the gonads is reassuring, a follow-up is necessary to determine the ovarian function of the patient. Thyroid function and glucose metabolism were analyzed in the context of previous reports of abnormalities of these parameters [16] and proved normal.

Pubertal development will be monitored in the future in order to induce puberty and permit normal bone mineralization. Should ovarian insufficiency be confirmed, oocyte cryopreservation should be considered. Hearing loss should be assessed and treated by a multidisciplinary team including an audiologist and otolaryngologist. Possible interventions for those with hearing loss include special educational resources, hearing aids, vibrotactile devices and cochlear implantation, which is an option for children older than 12 months with severeto-profound hearing loss [17]. In addition, due to highly intra- and inter-clinical variably, we tested her younger brother to exclude the presence of familial mutations and hence, the risk of hearing loss.

More recently, leukodystrophy was also associated with LARS2 pathogenic variants [15]. Concerning the development of neurological problems, systematic neurological surveillance is still not indicated, but it should be kept in mind that these may eventually develop later in life. More reports of older patients will help to elucidate the eventual progression of this syndrome.

Our case demonstrates that whole-exome sequencing is essential in the diagnosis of hearing loss in children. Benefits of receiving an early genetic diagnosis include the provision of prognostic information, streamlined care, accurate recurrence risk advice and, where appropriate, screening and treatment for associated health conditions.

\section{Abbreviations}

FSH: Follicle stimulating hormone; LH: Luteinizing hormone; $\mathrm{AMH}$ : AntiMüllerian Hormone

\section{Acknowledgements}

Not applicable.

\section{Authors' contributions}

MC examined the patient and the genetic data and was a major contributor in writing the manuscript. PK follows the patient in the endocrinology clinic and was a major contributor in writing the manuscript. SL performed and analyzed the genetic analysis and was a major contributor in writing the manuscript. MG performed and analyzed the genetic analysis and was a major contributor in writing the manuscript. MA was a major contributor in revising the manuscript. HCV follows the patient in ORL clinics and was a major contributor in writing the manuscript. NG follows the patient in ORL clinics and was a major contributor in writing the manuscript. AG examined the patient and the genetic data and was a major contributor in writing the manuscript. All authors read and approved the final manuscript.

\section{Funding}

No funding.

\section{Availability of data and materials}

All data generated or analyzed during this study are included in this published article. The mutations described in the current study are available in the ClinVar repository [ID: 55871 and 191173] https://www.ncbi.nlm.nih. gov/clinvar/variation/191173/ and https:/www.ncbi.nlm.nih.gov/clinvar/ variation $/ 55871 /$. The complete sequence datasets generated during the current study are not publicly available because individual privacy could be compromised.

Ethics approval and consent to participate

Written informed consent was obtained from patient's parents.

Consent for publication

Written informed consent was obtained from patient's parents.

\section{Competing interests}

The authors declare that they have no competing interests.

\section{Author details}

${ }^{1}$ Department of Genetic Medicine, University Hospitals of Geneva Rue, Gabrielle-Perret-Gentil 4, 1211 Genève 14, Switzerland. ²Departement of Pediatrics endocrinology, Geneva University Hospital, Geneva, Switzerland. ${ }^{3}$ Departement of ENT, Geneva University Hospital, Geneva, Switzerland.

Received: 6 September 2019 Accepted: 16 April 2020

Published online: 18 May 2020

\section{References}

1. Pallister PD, Opitz JM. The Perrault syndrome: autosomal recessive ovarian dysgenesis with facultative, non-sex-limited sensorineural deafness. Am J Med Genet. 1979;4(3):239-46.

2. Brodie EJ, Zhan H, Saiyed T, Truscott KN, Dougan DA. Perrault syndrome type 3 caused by diverse molecular defects in CLPP. Sci Rep. 2018;8(1): 12862.

3. Tracewska-Siemiątkowska A, Haer-Wigman L, Bosch D, et al. An expanded multi-organ disease phenotype associated with mutations in YARS. Genes (Basel). 2017;8(12):381.

4. Bullard JM, Cai YC, Spremulli LL. Expression and characterization of the human mitochondrial leucyl-tRNA synthetase. Biochim Biophys Acta. 2000; 1490(3):245-58.

5. Zerkaoui M, Demain LAM, Cherkaoui Jaouad I, Ratbi I, Amjoud K, Urquhart JE, O'Sullivan J, Newman WG, Sefiani A. Marfanoid habitus is a nonspecific feature of Perrault syndrome. Clin Dysmorphol. 2017;26(4):200-4.

6. Diodato D, Ghezzi D, Tiranti V. The mitochondrial aminoacyl tRNA synthetases: genes and syndromes. Int J of Cell Biol. 2014;2014:787956.

7. Kosaki R, Horikawa R, Fujii E, Kosaki K. Biallelic mutations in LARS2 can cause Perrault syndrome type 2 with neurologic symptoms. Am J Med Genet Part A. 2018;176(2):404-8

8. Pierce SB, Gersak K, Michaelson-Cohen R, Walsh T, Lee MK, Malach D, Klevit RE, King MC, Levy-Lahad E. Mutations in LARS2, encoding mitochondrial leucyl-tRNA synthetase, lead to premature ovarian failure and hearing loss in Perrault syndrome. Am J Hum Genet. 2013;92(4):614-20. 
9. Al-Jaroudi D, Enabi S, AlThagafi MS. Perrault syndrome with amenorrhea, infertility, Tarlov cyst, and degenerative disc. Gynecol Endocrinol. 2019; 35(12):1037-9.

10. Demain LAM, Urquhart JE, O'Sullivan J, Williams SG, Bhaskar SS, Jenkinson EM, Lourenco CM, Heiberg A, Pearce SH, Shalev SA, Yue WW, Mackinnon S, Munro KJ, Newbury-Ecob R, Becker K, Kim MJ, O'Keefe RT, Newman WG. Expanding the genotypic spectrum of Perrault syndrome. Clin Genet. 2017; 91(2):302-12.

11. Li Q, Wang K. InterVar: clinical interpretation of genetic variants by the 2015 ACMG-AMP guidelines. Am J Hum Genet. 2017;100(2):267-80.

12. Richards S, Aziz N, Bale S, Bick D, Das S, Gastier-Foster J, Grody WW, Hegde M, Lyon E, Spector E, Voelkerding K, Rehm HL. ACMG laboratory quality assurance committee. Standards and guidelines for the interpretation of sequence variants: a joint consensus recommendation of the American College of Medical Genetics and Genomics and the association for molecular pathology. Genet Med. 2015;17(5):405-24.

13. Riley LG, Rudinger-Thirion J, Schmitz-Abe K, Thorburn DR, Davis RL, Teo J, Arbuckle S, Cooper ST, Campagna DR, Frugier M, Markianos K, Sue CM, Fleming MD, Christodoulou J. LARS2 variants associated with Hydrops, lactic acidosis, Sideroblastic Anemia, and multisystem failure. JIMD Rep. 2016;28: 49-57.

14. Soldà G, Caccia S, Robusto M, Chiereghin C, Castorina P, Ambrosetti U, Duga S, Asselta R. First Independent Replication of the Involvement of LARS2 in Perrault Syndrome by Whole-Exome Sequencing of an Italian Family. J Hum Genet. 2016;61(4):295-300.

15. Van Der Knaap MS, Bugiani M, Mendes MI, Riley LG, Smith DEC, RudingerThirion J, Frugier M, Breur M, Crawford J, van Gaalen J, Schouten M, Willems M, Waisfisz Q, Mau-Them FT, Rodenburg RJ, Taft RJ, Keren B, Christodoulou J, Depienne C, Simons C, Salomons GS, Mochel F. Biallelic variants in LARS2 and KARS cause deafness and (ovario)leukodystrophy. Neurology. 2019; 92(11):e1225-37.

16. Lerat J, Jonard L, Loundon N, Christin-Maitre S, Lacombe D, Goizet C, Rouzier C, Van Maldergem L, Gherbi S, Garabedian EN, Bonnefont JP, Touraine P, Mosnier I, Munnich A, Denoyelle F, Marlin S. An application of NGS for molecular investigations in Perrault Syndrome: study of 14 families and review of the literature. Hum Mutat. 2016;37:1354-62.

17. Newman WG, Friedman TB, Conway GS, Demain LAM. Perrault Syndrome. 1993; In: Adam MP, Ardinger HH, Pagon RA, Wallace SE, Bean LH, Stephens K, Amemiya A, editors. Seattle (WA): University of Washington, Seattle; 19932020

\section{Publisher's Note}

Springer Nature remains neutral with regard to jurisdictional claims in published maps and institutional affiliations.

Ready to submit your research? Choose BMC and benefit from:

- fast, convenient online submission

- thorough peer review by experienced researchers in your field

- rapid publication on acceptance

- support for research data, including large and complex data types

- gold Open Access which fosters wider collaboration and increased citations

- maximum visibility for your research: over $100 \mathrm{M}$ website views per year

At $\mathrm{BMC}$, research is always in progress.

Learn more biomedcentral.com/submissions 